\title{
Automated Face Detection and Control System Using Computer Vision based Video Analytics to Avoid the Spreading of COVID-19
}

\author{
Charbel El Gemayel \\ TICKET Lab., Computer Sciences \\ Antonine University \\ Hadat-Baabda, Lebanon \\ charbel.gemayel@ua.edu.lb
}

\author{
Kabalan Chaccour, Member, IEEE \\ TICKET Lab., Computer \& Communications Eng. \\ Antonine University \\ Hadat-Baabda, Lebanon \\ NIT Lab., University of Bourgogne Franche-Comté \\ Belfort, France \\ kabalan.chaccour@ua.edu.lb
}

\author{
Joseph El Gemayel \\ Computer \& Information Sciences \\ University of Strathclyde \\ Glasgow, United Kingdom \\ joseph.el-gemayel@strath.ac.uk
}

\begin{abstract}
The classification of COVID-19 as global pandemic has led researchers and scientists to design solutions in order to reduce the fast spreading of the virus. This paper presents a novel detection and control system that utilizes Computer Vision based video analytics to help in reducing the speed of the spreading of the virus by recognizing people and detecting masks. The system uses the body temperature and other user biometrics to give access to a particular environement. The proposed system is able to identify a person who wants to access an environment and tracks his movement. The system can also control the door of the main entrance, the elevator, or any access zone, and generate audio notifications to alert user(s) to put their mask(s). The implementation results show that the proposed system has the advantages of a high sensitivity of $98.8 \%$ for front faces and 90.3\% for turned faces, and ensure a safe environment while preserving the benefits of being modular and low cost.
\end{abstract}

Index Terms-COVID-19, Digital Image Processing, Artificial Intelligence, Convolutional Neural Network, Face Detection

\section{INTRODUCTION}

Spread, severity and inaction are main elements that pushed the World Health Organization (WHO) to decide that COVID19 is classified as a global pandemic [14], [20]. The effect of this virus has impacted the entire world, changing the lives of human beings. It has affected the populations health, the capacity of healthcare systems, education, transportation, travel and tourism, and work environments.

IT technologies focused on different industries and integrated different types of digital surveillance cameras. The main reason behind this, is the decreasing rates of illegal violations and crimes. With recent advances in computing Hardware and Computer Vision (CV) Software, it is time to integrate these technologies into an automated control system. The Automated Control System will largely decrease the direct contact with people who don't wear masks. This will help in infection prevention and provide more care about the health of workers in different environments.

In this context, the current research proposes a complete Automated Control System that controls doors, panels, visible regions by sending commands in order to fight against
COVID-19. To our knowledge, the proposed approach is a new idea that has not been yet exploited. Our system architecture is automated, simple and easy to operate. The system can be easily implemented in many places such as malls, shops, cafes, public places, and many others where the environment has an IP cameras attached to the ceiling of each region or in front of main door or elevators. This system detects whether a person is wearing a mask or not, and it measures his temperature in order to open the main entrance door or to activate elevator panel. It also tracks the movement of people inside cameras covered areas in order to send sound voices when needed to alert the people to fix or wear the mask on their faces. The streaming videos from cameras are captured and analyzed using CV algorithms.

The rest of the paper is organized as follows. Section II describes the related work of most recent existing solutions. It gives a general overview on successful products, research techniques, algorithms that have already been deployed until now. Section III illustrates our proposed system approach that helps to control and protect our environment by analyzing all video streaming coming from all IP cameras. The section exposes the hardware and software parts of our proposed solution. Experiments are carried out in section IV where some drawbacks of the system are shown. We conclude our paper in section $\mathrm{V}$.

\section{RELATED WORK}

COVID-19 disease was declared a global pandemic by the WHO on 11 March 2020. In order to monitor and control the spread of this virus, a new technology must be applied. Artificial Intelligence (AI) can play this role and fight against it by producing notifications and suggestions about the infections control [2], [5]. Artificial intelligence with all its subsests, namely, Machine Learning (ML), Deep Learning [12], Natural Language Processing (NLP), and Computer Vision (CV) [22] are used with big data-based models for pattern recognition, explanation, speaking, translation, and prediction. 
Different methods with different algorithms have been implemented for face recognition during the past decade. Extracting face features or using a set of face images to compare it with the input data [6], [17], [18]. We can classify these detection methods into two groups as "KnowledgeBased Methods" and "Image-Based Methods". The first group uses information about facial features like using eyes, mouth, skin color or template matching in order to detect human faces. The other group uses training/learning methods to make comparison between face and non-face images. Examples of used methods are AdaBoost approach [23], [24], EigenFace [4], [13], [21] and Deep Learning Neural Networks (DLNN) [3], [7], [9], [10], [28], where we need to train the system on many images in order to increase its accuracy.

Automated monitoring systems are used to monitor and control activities, behaviors, changing data information of people, in the purpose of protecting, managing or directing them. CV helps to take this control and decisions, by being a good replacement of human visual ability. In surveillance systems, cameras are used for detecting or tracking objects, parking analysis, traffic detection or even crowd analysis [11], [15], [19]. Many researches have been conducted on aspects of face detection, such as identification, verification [16] or alone face detection [8], [27]. Using mask detection in such system plays a main role such as protecting from intruders [25], [26].

This paper seeks to design a real-time solution for an automated control system that reads streaming videos from multiple IP cameras connected to a network. The proposed software detects any face present in the field of view of the cameras, measures the temperature and detects the mask to take a decision by sending a dedicated notification. The system is running seamlessly without any connectivity with the user, easy to set it up, flexible, and extendable.

\section{Proposed System Approach}

Our proposed system approach upgrades the existing detection and controls systems using Artificial Intelligence. The system must have a modular architecture and configurable based on the application (e.g. access control, intrusion detection, fleet management, etc.). The proposed system must be reliable, easy to operate and implemented using low cost hardware and software. It should also benefits from the use of existing hardware such as Closed-Circuit TeleVision (CCTV) and access control systems. In the context of the new COVID19 pandemic, the aim is to prevent the spreading of the coronavirus inside premises such malls, shops, cafs, or public places. In this case, access to the premise is granted based on mask detection and body temperature.

\section{A. System Architecture}

The proposed system approach has a simple architecture (fig. 1). It is mainly composed of three parts namely, the sensing module, the remote processing module and the notification and control module. These modules are implemented using hardware and software components. In a typical access control scenario, when the user shows at the main entrance of a premise, his face is detected and recognized by the system and the door opens granting the user access to enter the premise. In the particular case of COVID-19 pandemic, access is not granted unless the user wears properly a mask on his face and has an appropriate body temperature.. Thus, when the user is in the proximity of the system sensors, his face is captured, and his temperature is measured. These data are fed to the remote processing system for more analysis. At this stage, CV based video analytics algorithms are applied to detect the mask on the user's face, measure the temperature and, if allowed, grant the access. Access is granted using the control and notification module. Each part of the system architecture is detailed in the following paragraphs.

1) Sensing module: The sensing module is the input to the system architecture. It is responsible for sensing and capturing data from the environment. It integrates different sensing functions that include proximity sensing, digital image capturing and temperature measurement. These functions are implemented using proximity sensors, digital cameras, and temperature sensors respectively. Temperature can be measured using Infrared (IR) remote sensing technology. One or more of these sensors can be installed depending on the location inside a premise. For instance, the entrance can be equipped with a proximity sensor, a temperature sensor and a camera. In another location, only a camera can be used to capture the face allowing by such different configurations of the system hardware. In this case, the premise can be divided into different zones where each zone can have its proper sensing module. Thus, each zone can benefit from particular analysis in the remote processing system. Moreover, the modularity of the proposed architecture allows the integration of existing detection and control systems. In fact, many premises have their private access control systems preinstalled. These systems use Radio Frequency Identification (RFID) readers, or biometric devices to grant access to users. RFID Readers for instance, extract the user ID engraved on badges or cards. These readers can be used as part of the sensing module where data from badges or cards can be combined with mask detection and temperature to grant the access to the user.

2) Remote processing system: The remote processing system is the second part of the system architecture. It constitutes the core of the architecture where our proposed video analytics model is running. It consists of a high processing computer or a server. From one side, the remote processing system connects to the sensing module via the Ethernet IP network. From the other side, it connects to the notification and control module through the WLAN interface.

Similarly, to the sensing module, the remote processing system can be an existing IP CCTV system where data from the IP cameras network are collected, stored and processed. More processing and analysis can be performed by applying advanced video analytics using AI depending on the application.

3) Control and notification module: The control and notification module is the output of the system architecture. It 
Sensing module

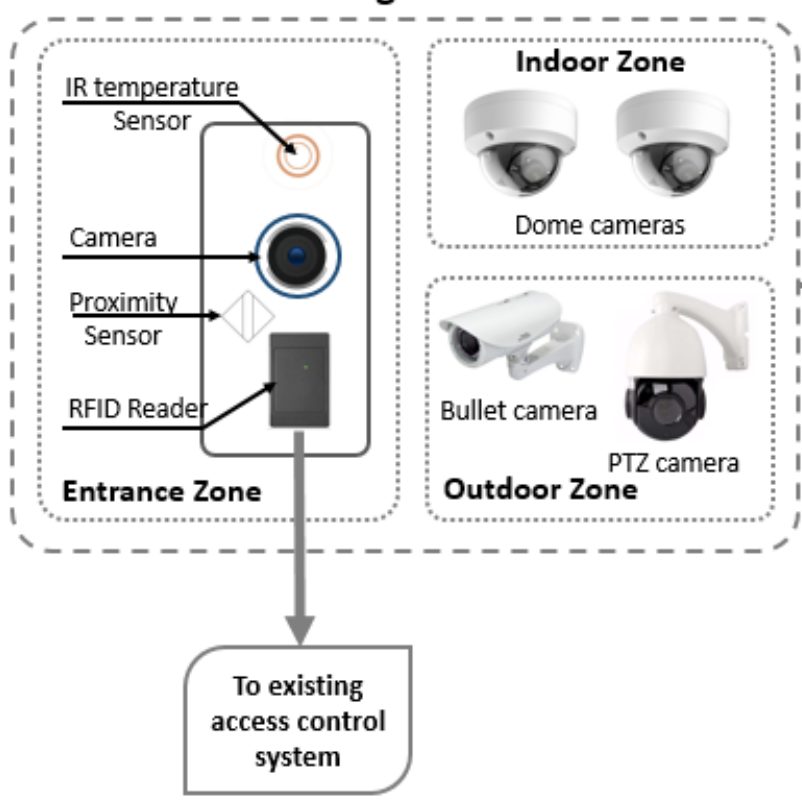

Remote processing system

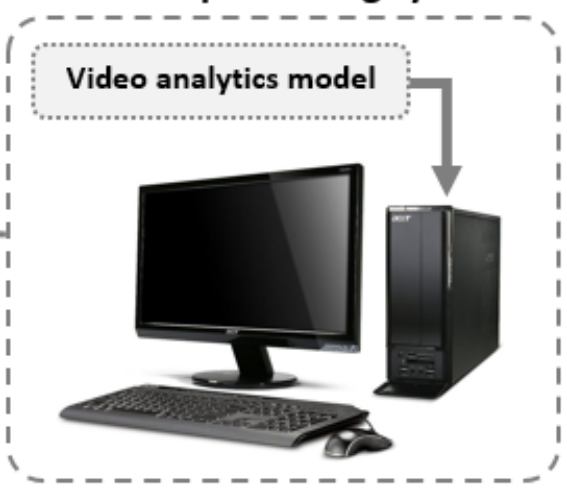

Control and notification module

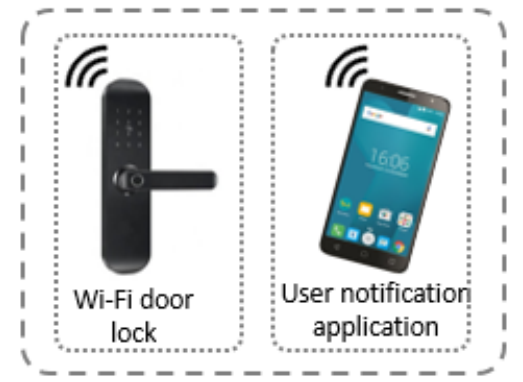

Fig. 1. Automated detection and control system architecture

integrates controlling and notification functions. These functions can be implemented by a hardware processing node or by a software application. The first can be designed with a compact Microcontroller Unit (MCU) with a Wi-Fi interface to control objects inside a premise such as a door lock or access to the lift. The second can be software application that runs on a smartphone, which notifies the user that he has to take a certain action, such as putting a mask.

\section{B. Proposed Video Analytics model}

The software part of the system architecture is the module where input data are processed and analyzed. The proposed model uses video analytics based on CV. This module is implemented on the remote processing system as illustrated in the architecture of Figure 1. The proposed model is configurable and runs in real time as a part of an IP-CCTV system. In the context of the current application of COVID-19 pandemic, the object is to detect the mask and recognize the user's face in premises where access restrictions are applied.

The input data of our proposed model are images captured from the cameras of the sensing module. Other input parameters such as temperature measurements are added to these images depending on the detection zone (e.g. main entrance). After that, images undergo a preprocessing phase where faces are extracted and fed to the CV algorithm for advanced analysis and recognition. The latter has been trained using a set of preprocessed images of the users. The outcome is assessed based on preset conditions (e.g. Mask or No Mask AND $36{ }^{\circ} \mathrm{C}<$ temp $<37{ }^{\circ} \mathrm{C}$ ). If the conditions are satisfied, the system commands the control and notification module to either grant or refrain access to the user. Figure 2 illustrates the main functional blocks of our proposed video analytics model. The following paragraphs provide the functional description of each block.

1) Preprocessing block: In this block, input data images from the cameras are captured and preprocessed. The preprocessing consists of extracting the faces from these images. Faces are extracted using OpenCV functions of the Free Cascade Classifier. This block has two working modes namely, the training mode and the normal mode.

- The training mode: In this mode, input data images are captured for the training set of our proposed model. A specific camera is used for this purpose. This camera is predefined by the system administrator. The process is as follows. The user is asked to sit in front of the camera. A set of images showing the user's face in different positions are captured. Then, the user is asked to put his mask on. Similarly, another set of images are captured showing the user's face with a mask. These two sets of images are labeled. Images with bare faces are labeled with ' 0 ' whereas images with faces having a mask are labeled with ' 1 '. Images with extracted faces and their labels are stored in a database of the analysis and recognition block for the training process. In addition, another set of random images are being fed for the training. This set is also used by the model to decide whether the user is wearing a mask or not. 


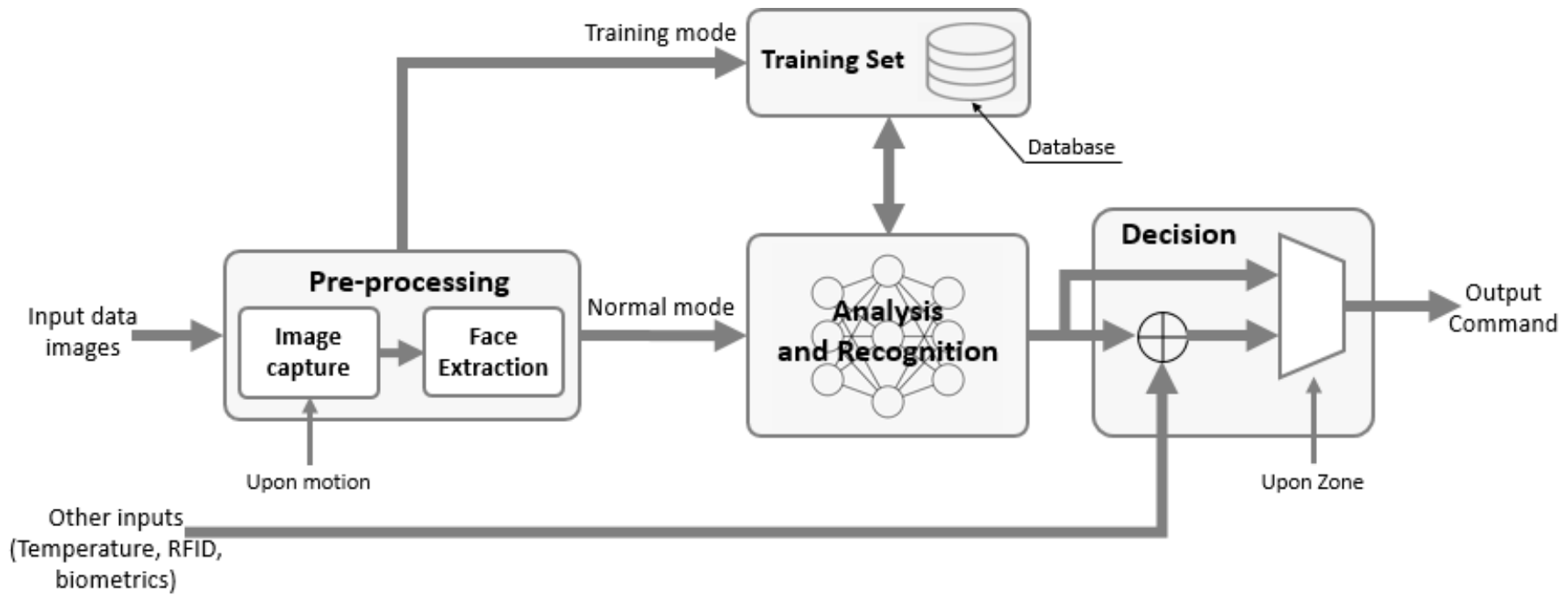

Fig. 2. Functional blocks of the proposed Video Analytics model

- The normal mode: In this mode, input data images are captured in real-time from cameras located in different zones. Whether at the main entrance of a premise or anywhere inside, images are captured upon motion. This is to reduce the storage capacity on the remote processing system. Users' faces are extracted from these images and fed to the block for analysis and recognition.

2) Analysis and recognition block: In this block, extracted faces from captured images are analyzed. The algorithm used in our model is the Convolutional Neural Network (CNN). The CNN is known at minimizing the loss and finding the right weights. When the system is in training mode, the model uses the images in the stored database for the training process. This includes the images of the extracted faces defined by the system administrator as well as the random set of images. When in normal mode, the images are fed directly to the model for analysis and recognition from the preprocessing block. These images are analyzed through the multiple layers of the neural network. The CNN is composed of 3 layers with a patch size of $8 * 8$ and generates a feature map with a depth of 64 as a batch size and 340 for the epoch parameter after many trials. The model has imput images of $640 * 640$ pixels. The output is a prediction of the user recognition and whether he is wearing a mask. Since the training set of images combine the users' faces with and without the mask, the system can recognize the user.

3) Decision block: In this block, the output command to the control and notification module is generated. This command is based on the prediction of the CNN algorithm which constitutes the input of this block. The output command is generated based on the zone where the user is detected. For instance, if the user is at the main entrance, the temperature and other user biometrics are added to generate the output command such as unlocking the main door. An audio notification is also generated that commands the user to put his mask on. On the other hand, when the detection happened in the other zones (e.g. input or output zones), the user will be notified through his smartphone, provided that the latter is connected to the WLAN of the premise.

\section{EXPERIMENTS AND RESULTS}

Our experiments reveal the robustness of our system. This section is divided into three parts namely, Experimental setup, Experimental scenarios, and Results and discussion.

\section{A. Experimental setup}

An object detector is designed to extract features from input images and then to feed these features through a prediction model that draws boxes around objects and predicts their classes. The model is the first object detector to connect the procedure of predicting bounding boxes with class labels in an end to end differentiable network.

Figure 3 gives a full functional description of the automated software. It is composed of three main processes namely, PreProcessing, Analysis \& Recognition, and Control \& Notification. During the first process, the captured image undergoes multiple steps. First the image is converted from RGB to grayscale to reduce the image variability, because luminance is by far more important in distinguishing visual features. Second, histogram equalization is applied to help adjust the contrast of the image in two different cases: dark or light background. Third, noise reduction removes color noise. This will help increase the focus on the detected object. And the fourth step, image resizing is applied. As known, less size gives less pixels which means less details. The second process consists of analyzing the preprocessed images in order to detect the mask. It is $\mathrm{CNN}$-based algorithm that aggregates and forms image features at different granularities. It consists of a series of layers to mix and combine image features to pass them forward for prediction. The output of the prediction and other parameters like the temperature of the body are handled in the third process. However, in this paper, we considered 


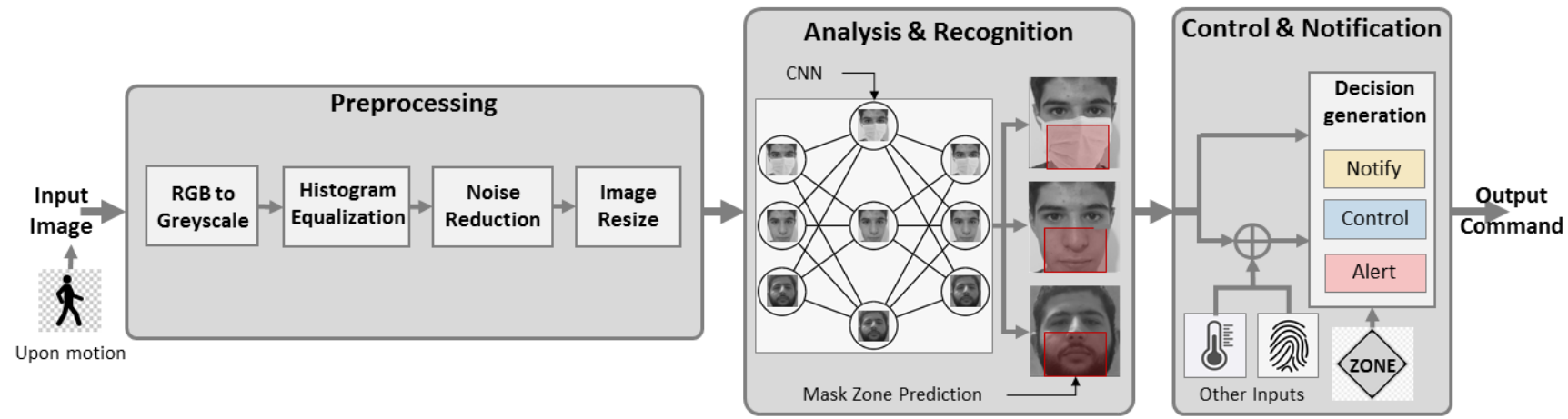

Fig. 3. Functional description of the proposed system software

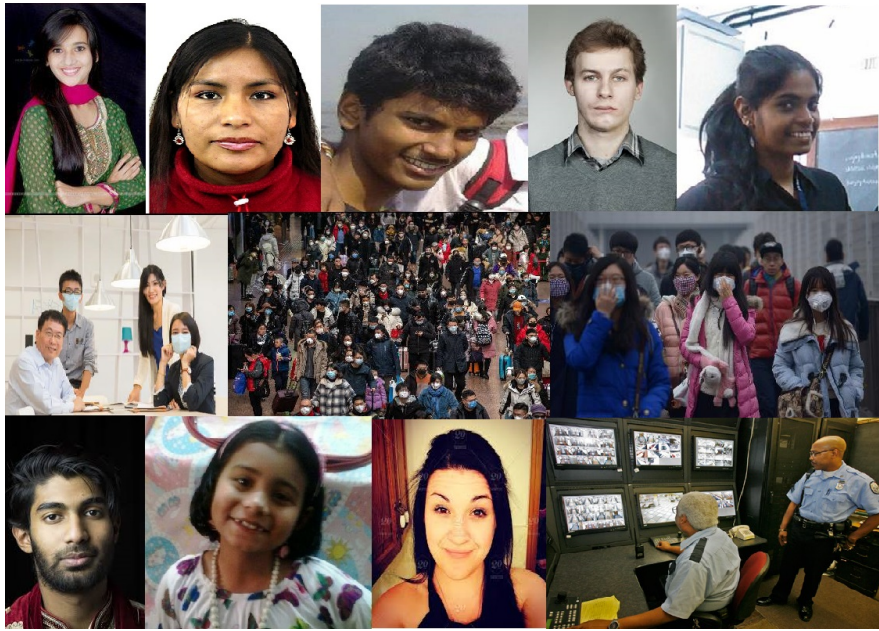

Fig. 4. Sample dataset of images

only the features related to face detection only. Other related parameter such as body temperature, biometrics and RFID will be used in the real-life deployement of the system. The control is automatic with different types of actions that can be taken: Start or Stop an alarm, Grant or Revoke access to a door, Generate an audible notification to the user, or Send a message to the remote administrator. The first and second processes are developed using Python, and the third process is developed with .Net framework.

\section{B. Experimental scenarios}

To validate our proposed video analytics model, experiments were carried on a set of random images. The dataset is composed of 3240 images of men and women including 1085 persons wearing masks and 2656 persons without masks. An example of the dataset images is shown in Figure 4. This dataset is used for training the model. For the results reported in this paper, approximately 650 people had their faces captured. A total of 1000 images for both front and turned faces were collected. The images were captured using a Milesight IR pro bullet network camera [1] in a real eviroment setup. The type is a surveillance camera that delivers up to $4 \mathrm{~K}$ resolution $(3840 * 2160$, effectively four times that of Full HD) at 30 frames per second (fps), providing users with ultra-highdefinition viewing experience. Its wide-angle lens, providing tall HD video stream, gives extended field-of-view both in horizontal and vertical directions.

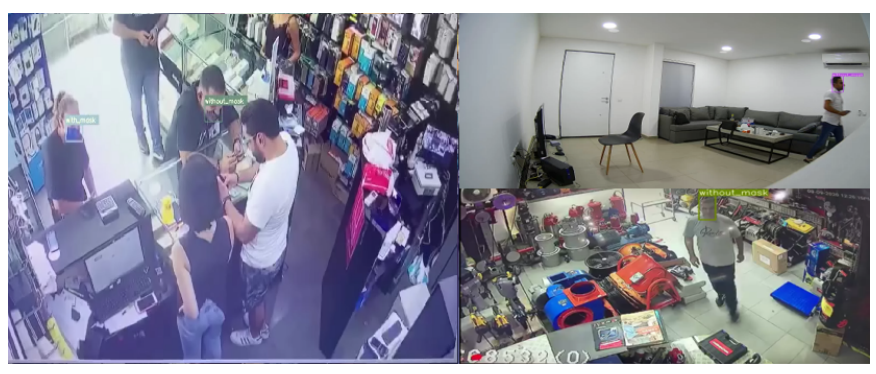

Fig. 5. Three different environment setups

The system is implemented into three different environments as shown in Figure 5. The first place is inside a house where the camera is fixed on a desk, and in two shops where the cameras were mounted on a ceiling. Noting that the left most image of Figure 5 shows the face detection with and without the mask.

\section{Results and Discussion}

The images are categorized into two groups: Front and Turned faces. The proposed algorithm achieves accuracy at detection rate of $98.8 \%$ with a false positive detection rate at $1.2 \%$. We tested two types of masks in our experiments: N95 respirators and surgical masks. The results for each category are presented in table I. The first row in the table shows the "Front Faces" category. The second row shows the "Turned Faces" detected to the left or right.

TABLE I

FACE DETECTION RESULTS

\begin{tabular}{|c|c|c|c|}
\hline $\begin{array}{c}\text { Image } \\
\text { Category }\end{array}$ & $\begin{array}{c}\text { \# Detected } \\
\text { Faces }\end{array}$ & $\begin{array}{c}\text { True Positive } \\
\text { Rate }\end{array}$ & $\begin{array}{c}\text { False Positive } \\
\text { Rate }\end{array}$ \\
\hline Front face & 500 & $98.8 \%$ & $1.2 \%$ \\
\hline Turned face & 500 & $90.3 \%$ & $4.1 \%$ \\
\hline
\end{tabular}




\section{CONCLUSION}

In this paper, we proposed an automated detection and control system that is designed to work with a conventional IP-CCTV system. The proposed model uses CV-based Video Analytics to detect whether the users are wearing their masks when they enter or roam inside premises such as universities, malls, etc. The aim is to reduce the spread of the COVID-19 inside these premises.

The proposed video analytics model is applied on input data images that are processed and analyzed to detect the mask zone on the people faces and generate the corresponding output commands. The model is validated using a set of random images with different types of masks. Detection results have shown high sensitivity (98.8\% for front faces and $90.3 \%$ for turned faces).

We are currently undertaking the implementation of our proposed model on an existing IP-CCTV system where the flow of images are generated from the network of IP cameras in real-time. The architecture is modified to match the proposed hardware described in Figure 1. The real-time processing of live streams and the location of the cameras are being investigated in our present activities and will be published in future papers.

Finally, the main objective of our system is to help reducing the speed of spread of Covid-19. However, it is worth mentioning that the system can be easily extended and adapted to different scenarios that require different rules such as the detection of other objects.

\section{REFERENCES}

[1] H.265* motorized pro bullet network camera.

[2] Zaheer Allam, Gourav Dey, and David S Jones. Artificial intelligence (ai) provided early detection of the coronavirus (covid-19) in china and will influence future urban health policy internationally. AI, 1(2):156165,2020

[3] Khairul Azha A Aziz, Ridza Azri Ramlee, Shahrum Shah Abdullah, and Ahmad Nizam Jahari. Face detection using radial basis function neural networks with variance spread value. In 2009 International Conference of Soft Computing and Pattern Recognition, pages 399-403. IEEE, 2009.

[4] Peter N. Belhumeur, Joao P Hespanha, and David J. Kriegman. Eigenfaces vs. fisherfaces: Recognition using class specific linear projection. IEEE Transactions on pattern analysis and machine intelligence, 19(7):711-720, 1997.

[5] Joseph Bullock, Alexandra Luccioni, Katherine Hoffman Pham, Cynthia Sin Nga Lam, and Miguel Luengo-Oroz. Mapping the landscape of artificial intelligence applications against covid-19. Journal of Artificial Intelligence Research, 69:807-845, 2020.

[6] Kelly A Gates. Our biometric future: Facial recognition technology and the culture of surveillance, volume 2. NYU Press, 2011.

[7] Kaiming He, Xiangyu Zhang, Shaoqing Ren, and Jian Sun. Delving deep into rectifiers: Surpassing human-level performance on imagenet classification. In Proceedings of the IEEE international conference on computer vision, pages 1026-1034, 2015.

[8] Erik Hjelmås and Boon Kee Low. Face detection: A survey. computer vision and image understanding. 1, 2001.
[9] Sergey Ioffe and Christian Szegedy. Batch normalization: Accelerating deep network training by reducing internal covariate shift. In International conference on machine learning, pages 448-456. PMLR, 2015.

[10] Alex Krizhevsky, Ilya Sutskever, and Geoffrey E Hinton. Imagenet classification with deep convolutional neural networks. Advances in neural information processing systems, 25:1097-1105, 2012.

[11] K Susheel Kumar, Shitala Prasad, Pradeep K Saroj, and Ramesh Chandra Tripathi. Multiple cameras using real time object tracking for surveillance and security system. In 20103 rd International Conference on Emerging Trends in Engineering and Technology, pages 213-218. IEEE, 2010.

[12] Yann LeCun, Yoshua Bengio, and Geoffrey Hinton. Deep learning. nature, 521(7553):436-444, 2015.

[13] AR Mohan and N Sudha. Fast face detection using boosted eigenfaces In 2009 IEEE Symposium on Industrial Electronics \& Applications, volume 2, pages 1002-1006. IEEE, 2009.

[14] World Health Organization et al. Novel coronavirus(2019ncov):situation report, 3. 1, 2020.

[15] Madhav Poddar, MK Giridhar, Amit Suresh Prabhu, V Umadevi, et al. Automated traffic monitoring system using computer vision. In 2016 International Conference on ICT in Business Industry \& Government (ICTBIG), pages 1-5. IEEE, 2016.

[16] Rajeev Ranjan, Swami Sankaranarayanan, Ankan Bansal, Navaneeth Bodla, Jun-Cheng Chen, Vishal M Patel, Carlos D Castillo, and Rama Chellappa. Deep learning for understanding faces: Machines may be just as good, or better, than humans. IEEE Signal Processing Magazine, 35(1):66-83, 2018.

[17] Henry Schneiderman and Takeo Kanade. A statistical method for $3 \mathrm{~d}$ object detection applied to faces and cars. In Proceedings IEEE Conference on Computer Vision and Pattern Recognition. CVPR 2000 (Cat. No. PR00662), volume 1, pages 746-751. IEEE, 2000.

[18] Frank Y Shih, Shouxian Cheng, Chao-Fa Chuang, and Patrick SP Wang. Extracting faces and facial features from color images. International Journal of Pattern Recognition and Artificial Intelligence, 22(03):515534, 2008.

[19] G Sreenu and MA Saleem Durai. Intelligent video surveillance: a review through deep learning techniques for crowd analysis. Journal of Big Data, 6(1):1-27, 2019.

[20] Paolo Surico and Andrea Galeotti. A user guide to covid19. London Business School. Available at: https://sites. google. com/site/paolosurico/covid-19, 1, 2020.

[21] CC Tsai, WC Cheng, Jin-Shiuh Taur, and Chin-Wang Tao. Face detection using eigenface and neural network. In 2006 IEEE International Conference on Systems, Man and Cybernetics, volume 5, pages 43434347. IEEE, 2006.

[22] Anwaar Ulhaq, Asim Khan, Douglas Gomes, and Manoranjan Pau. Computer vision for covid-19 control: A survey. arXiv preprint arXiv:2004.09420, 1, 2020.

[23] Paul Viola and Michael Jones. Rapid object detection using a boosted cascade of simple features. In Proceedings of the 2001 IEEE computer society conference on computer vision and pattern recognition. CVPR 2001, volume 1, pages I-I. IEEE, 2001.

[24] Paul Viola and Michael J Jones. Robust real-time face detection. International journal of computer vision, 57(2):137-154, 2004.

[25] Weitao Wan and Jiansheng Chen. Occlusion robust face recognition based on mask learning. In 2017 IEEE International Conference on Image Processing (ICIP), pages 3795-3799. IEEE, 2017.

[26] Che-Yen Wen, Shih-Hsuan Chiu, Yi-Ren Tseng, and Chuan-Pin Lu. The mask detection technology for occluded face analysis in the surveillance system. Journal of Forensic Science, 50(3):1-9, 2005.

[27] Stefanos Zafeiriou, Cha Zhang, and Zhengyou Zhang. A survey on face detection in the wild: past, present and future. Computer Vision and Image Understanding, 138:1-24, 2015.

[28] Cha Zhang and Zhengyou Zhang. A survey of recent advances in face detection. 1, 2010. 